\title{
MANO ROBÓTICA COMO ALTERNATIVA PARA LA ENSEÑANZA DE CONCEPTOS DE PROGRAMACIÓN EN ARDUINO
}

\section{ROBOTIC HAND AS ALTERNATIVE FOR TEACHING OF PROGRAMMING CONCEPTS IN ARDUINO}

\author{
Jorge Armando Niño Vega, Lina Yormary Martínez Díaz \\ PhD. Flavio Humberto Fernández Morales \\ Universidad Pedagógica y Tecnológica de Colombia - UPTC \\ Sede Duitama, Colombia, Tel: (+578) 7428263. \\ E-mail: jorge-258.1@hotmail.com, linayormary.martinez@uptc.edu.co, \\ flaviofm1@gmail.com
}

\begin{abstract}
Resumen: En este trabajo se presenta el desarrollo de una mano robótica, explicando cuales son las partes principales que conforman el prototipo. El diseño se realizó en AutoCAD y SolidEdge, programas que permitieron dimensionar la estructura mecánica. El control electrónico se efectuó a través de una tarjeta Arduino, la cual permite leer el estado de los sensores, flexorresistencias en este caso, a la vez que genera las señales para controlar los servomotores responsables del movimiento. En una primera aproximación se construyó una versión de bajo costo, con materiales reciclados, la cual permitió validar el diseño propuesto. La versión final se construyó con materiales disponibles comercialmente, dando como resultado un prototipo didáctico que puede ser utilizado como herramienta motivadora para el aprendizaje de programación en Arduino.
\end{abstract}

Palabras clave: Mano robótica, Arduino, programación, enseñanza de tecnología.

\begin{abstract}
In this paper, the development of a robotic hand is described, explaining which are the main parts that conform the prototype. The design was made in AutoCAD and SolidEdge, software that allowed sizing the mechanical structure. Electronic control was made through an Arduino card that permits reading the sensor's state, in this case flexoresistences, and generating signs to control the servomotors that are responsible of movement. In the first approach, a low-cost, recycled-material version was built and it allowed validating the proposed design. The final version was made with commercially available materials, giving as result a teaching prototype that can be used as motivational tool for learning of programming in Arduino.
\end{abstract}

Keywords: Robotic hand, Arduino, programming, technology teaching.

\section{INTRODUCCIÓN}

La robótica es una de las áreas más importantes de la ingeniería moderna ya que provee dispositivos automatizados, que incrementan el rendimiento de los procesos industriales (Gutiérrez-Ríos, Martínez-Oviedo, \& Peña-Cortés (2013). Esto se refleja en la gran cantidad de aplicaciones relacionadas con el control de posición, visión artificial, sistemas de clasificación y medición de parámetros físicos, por mencionar algunas (TorresBarahona, León-Medina, \& Torres-Díaz 2012; Cárdenas \& Prieto-Ortíz 2015; Velazco-Cáceres \& Pinto-Salamanca, 2012; Carreño-Bodensiek, 2010).

Estas aplicaciones exigen en los desarrolladores una gran capacidad para programar, siendo necesario el conocimiento de estructuras, lógica y lenguajes de programación, junto con el manejo de 
técnicas de control y procesamiento de señales (León-Medina \& Torres-Barahona, 2015). En este sentido es deseable que los ingenieros, desde su formación preuniversitaria, se aproximen a la programación en contextos didácticos, que les permitan apropiar competencias que luego serán muy útiles en el ejercicio profesional.

En Colombia, el Ministerio de Educación Nacional ha establecido el área de tecnología e informática como el espacio formativo para que los estudiantes apropien conocimientos relacionados con los principios de funcionamiento y uso de artefactos (Angarita-Velandia, Fernández-Morales, \& Duarte, 2011). Muchas instituciones educativas han tomado a la informática y en particular a la programación en la plataforma Arduino, como alternativa para brindar la formación en tecnología (Angarita-Velandia, Fernández-Morales, \& Duarte,2014). Arduino, sistema para desarrollo con microcontroladores de bajo costo, es una herramienta fácil de utilizar y se puede convertir en una ayuda didáctica en el aula (Delgado, Güell, García, Conde, \& Casado, 2014).

La falta de ayudas didácticas para que los estudiantes plasmen sus ideas sobre lo que se está aprendiendo en programación, ha sido un gran problema en el desarrollo de las competencias del área de tecnología. Lo anterior se une a la poca capacitación de los docentes, lo cual genera inconvenientes a la hora de llevar los contenidos al aula (Parra-León, Duarte, \& Fernández-Morales, 2014). De lo anterior surge la necesidad de implementar una ayuda didáctica que motive el aprendizaje de programación con Arduino, facilitando la orientación de la temática por parte de los docentes.

En este contexto, la aplicación de la robótica en el campo educativo ha venido creciendo, gracias a la versatilidad de estos dispositivos, y se constituye en una herramienta de motivación para el aprendizaje de temáticas relacionadas con tecnología (García-Hurtado, Castillo-García, \& Escobar-Jiménez, 2012). Adicionalmente, la robótica se ha empleado para afianzar las destrezas en la solución de problemas en los niveles de educación básica y media, así como en el desarrollo de habilidades de proceso científico en la formación inicial de docentes e ingenieros, entre otros profesionales (Monsalves-González, 2011; Reyes-González, \& García-Cartagena, 2014).

La robótica educativa implica el desarrollo de prototipos reales, permitiendo la aplicación creativa del conocimiento de los estudiantes (Bravo-Sánchez \& Forero-Guzmán, 2012). Estos prototipos son una herramienta importante para comprender conceptos abstractos y complejos, a la vez que permiten el desarrollo de competencias básicas como el trabajo en equipo (Moreno et al., 2012). Aunque existen diversas alternativas, como los seguidores de luz y los robots inspirados en insectos (Mesa-Mesa \& Barrera-Lombana, 2013), una opción a explorar es la mano robótica, la cual puede constituirse en un prototipo didáctico que motive a los estudiantes en el aprendizaje de la temática de programación (Ponson, 2013).

En este trabajo se plantea el desarrollo de una mano robótica como prototipo didáctico, a través del cual el estudiante podrá acceder de manera interactiva a las temáticas propias de la programación en Arduino. De esta forma se espera incrementar la motivación de los estudiantes por el área de tecnología, a la vez que se mejoran las competencias de programación. A continuación, se presenta el diseño propuesto, junto con detalles del proceso de ensamble y puesta a punto.

\section{MATERIALES Y MÉTODOS}

\subsection{Diseño propuesto}

El principal objetivo pedagógico de la mano robótica es el de permitir al estudiante interactuar con ella, identificando la funcionalidad de cada uno de sus componentes, a la vez que motiva al aprendizaje de los temas relacionados con programación. Desde el punto de vista técnico, el prototipo deberá permitir la movilidad de los cinco dedos, emulando el cierre y apertura de las falanges, movimiento que es dado por el usuario a través de un guante.

La figura 1 presenta la estructura propuesta para la mano robótica, la cual se basa en seis bloques funcionales, a saber: mando, control, conexión, sensorial, control de movimiento y el bloque de movimiento.

La mano robótica se programa a través de Arduino, permitiendo la simulación del movimiento de los cinco dedos, controlada a través de un guante sensorial. Arduino, sistema para desarrollo con microcontroladores de bajo costo, es una plataforma de hardware y software libre, factible de usar en diversas aplicaciones, especialmente en las de adquisición y procesamiento de señales (Gausin, 2012). Aunque existen otros sistemas de desarrollo, como los basados en 
microcontroladores PIC de Microchip (FernándezMorales \& Duarte, 2012), se escogió Arduino debido a que cuenta con un entorno de programación gráfica y descriptiva muy simple, adecuado para la enseñanza en los primeros niveles educativos.

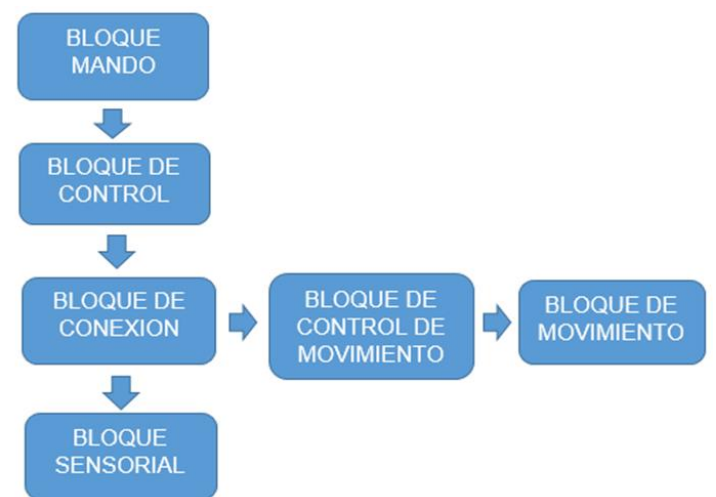

Fig. 1. Diagrama en bloques de la mano robótica didáctica.

En la figura 1 se observan varias etapas o bloques, los cuales se relacionan entre sí para el correcto funcionamiento de la mano robótica. En primer lugar, se tiene el bloque de mando, el cual corresponde al software ARDUINO, en donde el estudiante programa al bloque de control, teniendo en cuenta las variables de entrada y salida. Luego se encuentra el bloque de control, donde el estudiante realiza las conexiones en la placa Arduino, conectando los elementos intangibles con los artefactos tangibles, mediante el programa que se realiza en el bloque de mando.

Después del bloque de control, en la figura 1 se encuentra el bloque de conexión y de este se ramifican el bloque de control de movimiento y el bloque de movimiento. La característica importante de estos bloques es que en ellos el estudiante realiza las conexiones en el Protoboard de los diferentes componentes, como: servomotores, flexorresistencias y leds, entre otros. Además, el bloque de control de movimiento utiliza las señales que se generan en el software Arduino, para posicionar los servomotores a su estado inicial.

El bloque de movimiento está constituido por el mecanismo que conforma la estructura física de los dedos de la mano robótica, los cuales responden a las señales del bloque de control de movimiento. Por último, en el bloque sensorial el estudiante realiza la conexión de las flexorresistencias en el guante; esto para que, al ponérselo y flexionar los dedos, el prototipo didáctico replique los movimientos que el estudiante esté realizando, de la manera más óptima y eficiente posible.

\subsection{Metodología}

El desarrollo del prototipo implica el diseño de una estructura mecánica, la cual brinda el soporte físico. En este caso se utilizaron AutoCAD y SolidEdge, programas que permitieron dimensionar la estructura mecánica.

La estructura de cada dedo se implementó con una manguera de PVC flexible de $23 \mathrm{~mm}$ de diámetro, con un refuerzo de PVC rígido en espiral antichoque, que soporta una presión de 150 psi. Esta manguera se une a un servomotor a través de un Nylon, hilo de FluoroCarbón transparente, de 0,30 $\mathrm{mm}$ de diámetro y resistencia de $12,62 \mathrm{Kg}$. Los dedos se unen a una caja de madera de $3 \mathrm{~mm}$ de espesor, que representa la palma de la mano; en ella se encuentran 3 LEDs, diodos emisores de luz, que indican si el guante sensorial está abierto o cerrado.

Los dedos son movidos por cinco servomotores SG90, con un torque de $1,98 \mathrm{~kg}-\mathrm{cm}$ a $4,8 \mathrm{~V}$ y con un ángulo de rotación de $180^{\circ}$. Además, se emplearon 5 flexorresistencias SEN-10264, las cuales se adaptaron a un guante de Nylon con palma de Nitrilo. El componente electrónico se implementó en una placa de Arduino UNO-R3, la cual se programa a través del software Arduino IDE, Integrated Development Environment por sus siglas en inglés (Enríquez-Herrador, 2009).

La placa de Arduino UNO R3 incorpora un microcontrolador ATmega328, el cual cuenta con 14 pines de entrada y salida digitales, de los cuales 6 son salidas PWM; además, posee 6 entradas analógicas. En este caso, el prototipo requiere de cinco entradas analógicas para las flexorresistencias, una salida digital para el funcionamiento de las cargas lumínicas y cinco pines PWM para el control de los servomotores.

\section{RESULTADOS Y DISCUSIÓN}

\subsection{Estructura mecánica}

En la figura 2 se observa la simulación de la estructura mecánica de la mano robótica, realizada en Solid Edge, en donde se referencian: la posición de los dedos, los LEDs y los servomotores. También se aprecia el hilo que se ata a cada servomotor y a cada dedo, el cual brinda la tensión mecánica necesaria para mover la estructura. 


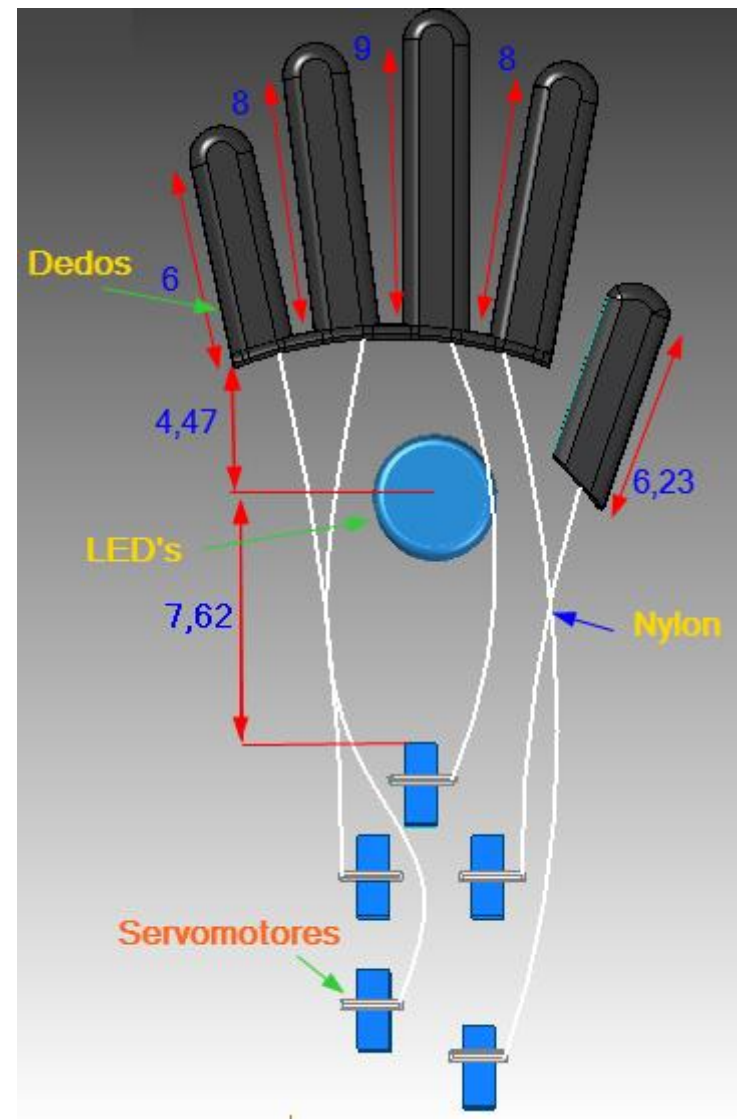

Fig. 2. Estructura mecánica de la mano robótica visualizada en Solid Edge.

Igualmente, en la figura 2 se aprecian las dimensiones de cada dedo y la separación que hay entre los servomotores, los cuales se ubicaron de modo que al energizarlos no se choquen entre sí, previniendo que se enreden los hilos.

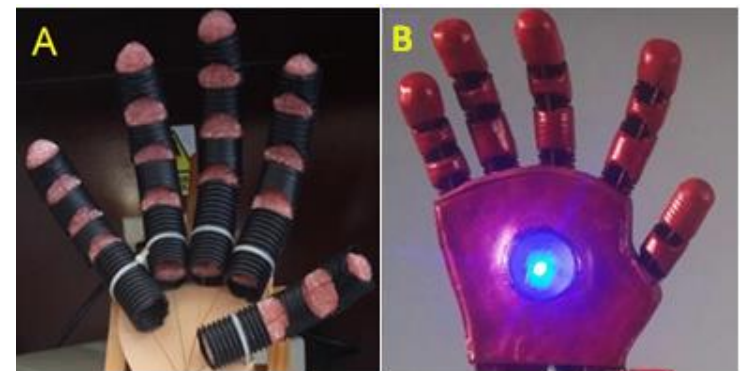

Fig. 3. Fases de construcción de la estructura de los dedos.

En la figura 3 se muestran la construcción de la estructura de los dedos. La sección A, indica que los dedos están compuestos por cinco segmentos de manguera de PVC flexible; en cada segmento se insertan pequeñas porciones de espuma, con el fin de que la estructura retorne a su posición inicial una vez que se deja de tensionar el hilo que la hace flectar; inicialmente, para facilitar el ensamble, la estructura se encuentra asegurada a una tabla por medio de abrazaderas.

Los dedos se recubren con caucho de silicona; este material permite la torsión de los dedos, a la vez que se previenen rupturas en la manguera de PVC; la estructura se incrusta en un molde hecho en madera, el cual brinda estabilidad al armazón. En la figura $3 \mathrm{~B}$ se observa el acabado de la estructura, para el cual se emplea porcelanicrón y pintura metalizada en aerosol, con el fin de incrementar la dureza y mejorar la presentación.

\subsection{Detalle de los bloques funcionales}

En la figura 4 se aprecia el montaje de todos los componentes electrónicos de la mano robótica. En primer lugar, se tiene la fuente de alimentación DC, una batería de Li en éste caso, la cual alimenta un regulador de $5 \mathrm{~V}$, tensión de funcionamiento de los circuitos. También se tiene la placa de Arduino UNO R3, la cual procesa la información enviada desde la IDE a través del cable de comunicación; a esta placa se conectan las entradas analógicas de las flexorresistencias y las salidas digitales de los servomotores.

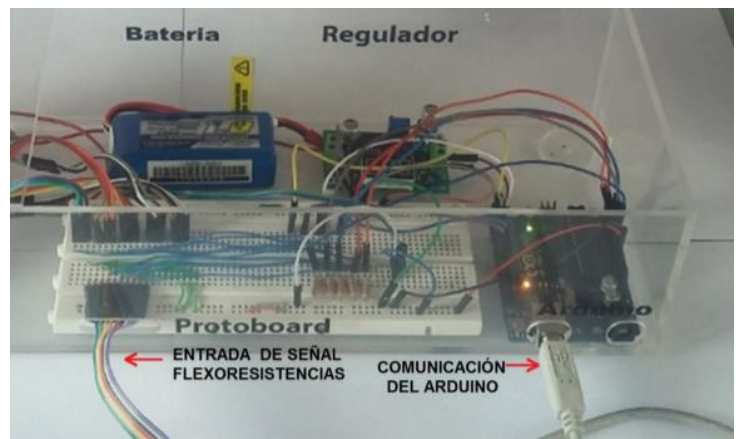

Fig. 4. Montaje electrónico de la mano robótica.

El montaje electrónico se encuentra instalado dentro de una caja de acrílico de $3 \mathrm{~mm}$ de espesor, donde se ha demarcado la ubicación de cada componente para su fácil identificación; allî también se ubica el interruptor para energizar el prototipo. El protoboard corresponde al bloque de Conexión, en el cual el estudiante realiza la conexión de los servomotores, las flexorresistencias, los LEDs y energiza la mano robótica.

El bloque de movimiento está constituido por un mecanismo que conforma la estructura física de los dedos de la mano robótica, los cuales responderán al movimiento de los servomotores, como se aprecia en la figura 5 . 


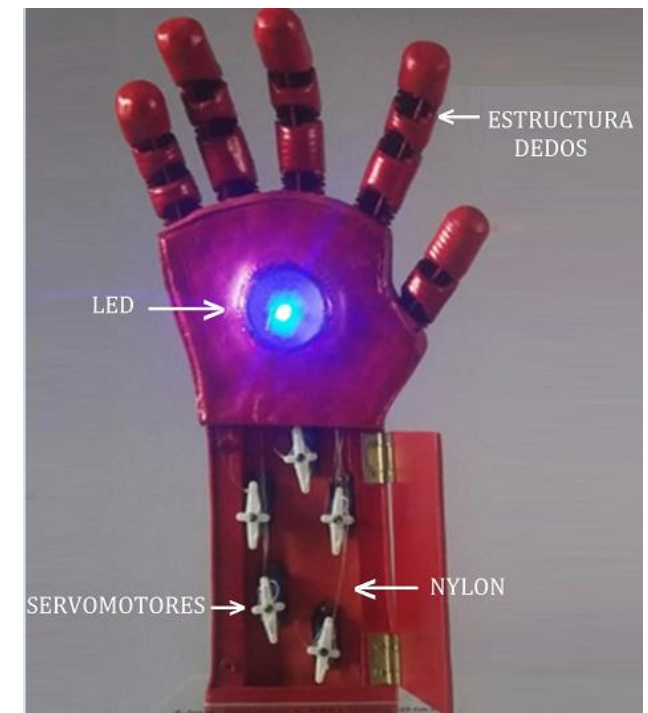

Fig. 5. Bloque de control de la mano robótica didáctica.

En la figura 5 se observa la estructura donde se emula el movimiento de los dedos, los LEDs que indican la apertura y cierre de la mano, junto con los cinco servomotores incrustados en una caja de madera que puede ser accesada por los estudiantes.

Las flexorresistencias se montan como se muestra en la figura 6; estos elementos responden a la cantidad de curvatura a la que se someten, generando un valor de resistencia eléctrica variable. Cuando el estudiante mueve los dedos en el guante, las flexorresistencias detectan el movimiento y lo traducen en información que emplea el bloque de mando para generar los pulsos requeridos por los servomotores para replicar el movimiento.

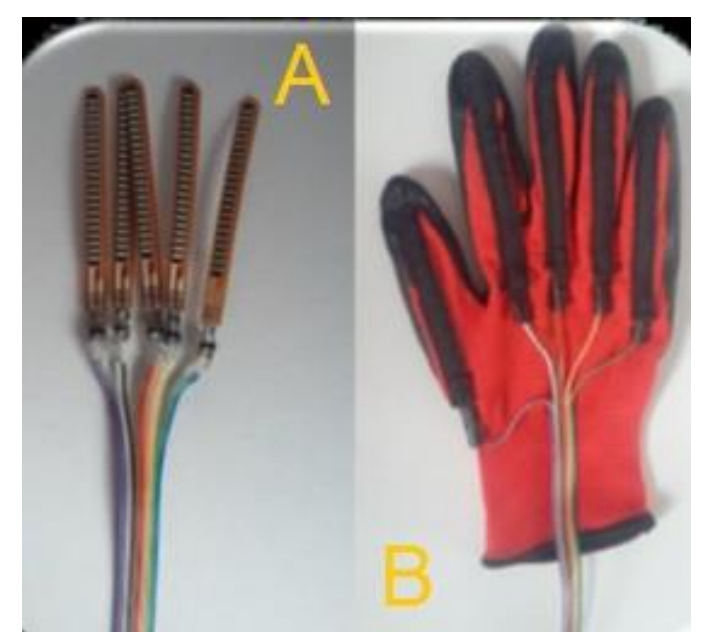

Fig. 6. Guante sensorial de la mano robótica didáctica.
En la figura 6 A se aprecian las cinco flexorresistencias que se encuentran soldadas a un cable ribon de $60 \mathrm{~cm}$ de largo. En la figura $6 \mathrm{~B}$ se observa un guante de nylon con palma de nitrilo, al cual se han cocido en las falanges cinco fundas, una por cada dedo, en las cuales se insertan las flexorresistencias.

\subsection{Prototipo resultante}

Hasta el momento se han realizado dos versiones del prototipo. En una primera aproximación se emplearon materiales reciclados, de bajo costo, con flexorresistencias de fabricación artesanal -papel, grafito y papel aluminio-. En la figura 7 se identifican la estructura de la mano y el guante sensorial, el cual es de lana.

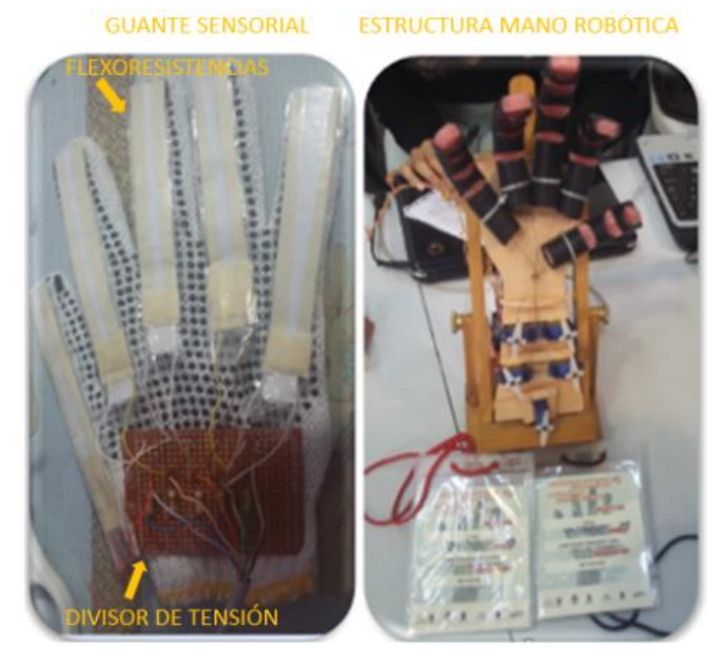

Fig. 7. Primera versión del prototipo.

La primera versión del prototipo permitió establecer la funcionalidad del diseño. Sin embargo, la interacción con los estudiantes causó el deterioro del dispositivo, esto sin contar con la falta de precisión en los movimientos de los dedos. En vista de lo anterior, se elaboró una segunda versión del prototipo, ver figura 8 .

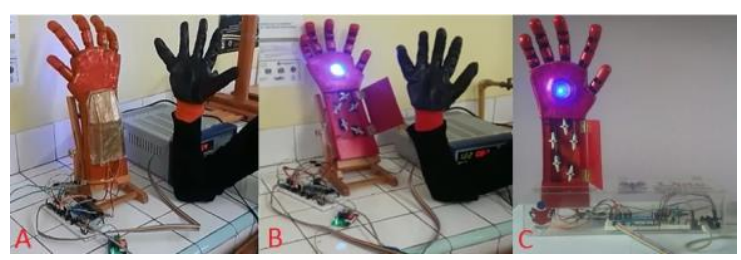

Fig. 8. Versión funcional del prototipo.

En la figura 8 se presenta el prototipo final: en la sección A se muestra la parte posterior de la mano, con un diseño atractivo para los estudiantes; en la sección B se ilustra la parte frontal de la mano robótica; en la sección $\mathrm{C}$ se aprecia el prototipo 
final junto con la caja de conexión y guante sensorial. En la segunda versión de la mano robótica se emplearon flexorresistencias comerciales, lo cual permitió mejoras en la precisión. Igualmente, se emplearon materiales de mejor calidad, los cuales brindan mayor durabilidad al prototipo.

La figura 9 ilustra la programación para el funcionamiento de uno de los dedos de la mano robótica. La calibración en esta etapa es un punto importante para el buen funcionamiento del dispositivo, ya que los servomotores deben ser llevados a posiciones angulares específicas. Al enviar una señal codificada, la posición angular de los servomotores cambia y se mantendrá así mientras exista una línea de entrada; esto ocurre siempre que las flexorresistencias estén flectadas.

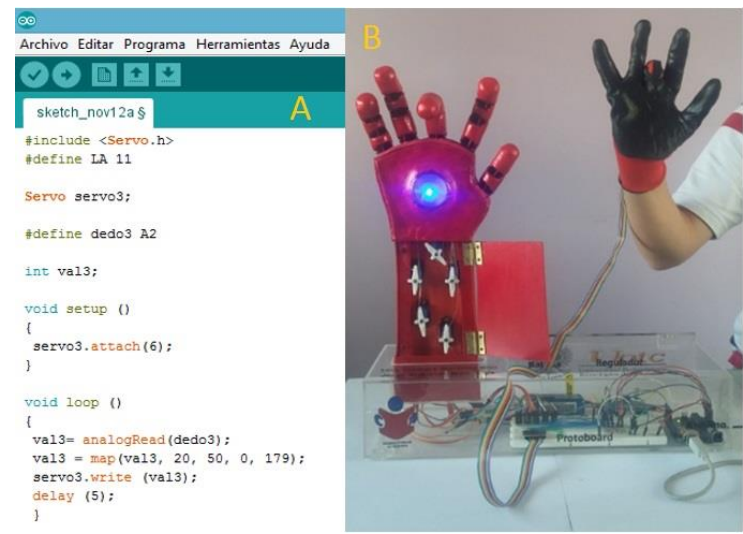

Fig. 9. Operación con el dedo corazón.

En la figura 9 A se identifica la programación del dedo corazón; en ella se incluye la librería para el manejo de los servomotores, se definen los pines a utilizar y el mapeo para relacionar los valores de las flexorresistencias con los ángulos del servomotor. En la figura 9 B se observa a la mano robótica siguiendo el movimiento del dedo corazón realizado por un usuario.

Al alimentar el bloque sensorial y manipular el guante, los sensores flexibles envían una señal que depende del grado de flexión, cuya resistencia incrementa de acuerdo al grado de inclinación de la misma. Al variar la inclinación de los sensores se obtiene un cambio en la posición de inicio de los servomotores, haciendo que estos simulen el movimiento que realiza la persona que manipula el guante, tal y como se observa en la figura $9 \mathrm{~B}$.

Desde el punto de vista técnico, se obtuvo un prototipo de mano robótica que permite los siguientes movimientos: al hacer flexión a las flexorresistencias, variará el ángulo de posicionamiento de los servomotores y, a su vez, cada dedo se moverá; cuando no haya flexión, el dedo estará a $10^{\circ}$ de inclinación con respecto a la posición de máxima elongación; esto se debe a la deformación inicial de la estructura por su propio peso. Cuando el guante esté completamente cerrado, los dedos llegarán a su máxima flexión, con una posición de $90^{\circ}$ respecto a su posición inicial.

Como resultado del diseño descrito anteriormente, se obtuvo un equipo portátil, llamativo y fácil de utilizar, lo cual ayuda al trabajo de los docentes a la hora de utilizarlo como material didáctico para la enseñanza de programación en Arduino, en instituciones de educación básica y media.

El proyecto se complementa con un manual de usuario, donde se brindan las indicaciones para la conexión de los componentes. Igualmente, se sugieren prácticas en algunas temáticas básicas como: estudio del funcionamiento de diferentes componentes electrónicos; circuitos eléctricos básicos: serie, paralelo y mixto; programación de entradas y salidas digitales, así como la programación de entradas análogas, en la IDE de Arduino.

La práctica de aula se realizará con el apoyo de una plataforma de administración de contenidos, LMS por sus siglas en inglés, a través de la cual el estudiante podrá acceder a información complementaria y resolver dudas en foros programados específicamente sobre la temática. De esta manera se busca integrar a las Tecnologías de la Información y la Comunicación, TIC, para promover la transformación pedagógica que potencie procesos de interacción, autonomía, el aprender a aprender y la participación activa de los estudiantes en su proceso formativo (Garcés-Pretel, Ruiz-Cantillo, \& Martínez-Ávila, 2014).

La realización de una mano robótica como prototipo didáctico, a diferencia de otros prototipos como: seguidor de luz, seguidor de línea, maquetas de ascensores o semáforos, tiene como ventaja el ser poco común, resultando así un artefacto más llamativo para el estudiante.

\section{CONCLUSIONES}

El diseño y construcción de la mano robótica didáctica permitió establecer la viabilidad técnica y pedagógica del prototipo. Igualmente se evidenció 
la posibilidad de fabricar un dispositivo de bajo costo, el cual puede ser replicado fácilmente por los estudiantes.

Sin embargo, para garantizar la durabilidad del prototipo, así como la precisión en los movimientos, se implementó una segunda versión de la mano robótica, la cual es adecuada para la interacción continua con los usuarios.

El prototipo se ha presentado en algunas muestras de ciencia y tecnología, donde despertó el interés por parte de estudiantes y docentes, al querer entender cómo fue su modo de construcción, programación y conexión. Las pruebas preliminares indican que para los niños pequeños es llamativo el movimiento que realiza la mano robótica debido a que les resulta semejante al de una mano real.

La mano robótica fue diseñada y construida de tal modo que los estudiantes pueden interactuar con ella de forma segura, permitiendo el acceso a los diversos componentes, lo cual resalta el aspecto didáctico del prototipo. Adicionalmente, el prototipo permite abordar temáticas como: el software Arduino, activación de LEDs, programación de servomotores y adquisición de información a través de sensores, entre otras.

Como trabajo futuro se tiene la aplicación de la mano robótica en el aula, para lo cual se espera utilizar una plataforma de administración de contenidos, donde se ubicará la guía de actividades y material complementario como videos y enlaces a páginas de interés.

\section{REFERENCIAS}

Angarita-Velandia, M. A., Fernández-Morales, F. H., \& Duarte, J. E. (2011). Utilización de material didáctico para la enseñanza de los conceptos de ciencia y tecnología en niños. Revista de Investigación, Desarrollo e Innovación, 2 (1), 35-43. Recuperado de: http://revistas.uptc.edu.co/revistas/index.php/in vestigacion_duitama/article/view/1307

Angarita-Velandia, M. A., Fernández-Morales, F. H., \& Duarte, J. E. (2014). La didáctica y su relación con el diseño de ambientes de aprendizaje: una mirada desde la enseñanza de la evolución de la tecnología. Revista de Investigación, Desarrollo e Innovación, 5 (1), 46-55. https://doi.org/10.19053/20278306.3138
Ponson, M. (2013). HackerHAND: la mano robótica que busca acercar la tecnología a los escolares. Recuperado de http://facultades.unab.cl/ingenieria/2013/08/05/ hackerhand-la-mano-robotica-que-buscaacercar-la-tecnologia-a-los-escolares/

Bravo-Sánchez, F. A., \& Forero-Guzmán, A. (2012). La robótica como un recurso para facilitar el aprendizaje y desarrollo de competencias generales. Revista Teoría de la Educación: Educación y Cultura en la Sociedad de la Información, 13 (2), 120-136. Recuperado

de http://www.redalyc.org/pdf/2010/20102439000 7.pdf

Cárdenas, J. A., \& Prieto-Ortíz, F. A. (2015). Diseño de un algoritmo de corrección automática de posición para el proceso de perforado PCB, empleando técnicas de visión artificial. Revista de Investigación, Desarrollo e Innovación, 5 (2), 107-118. doi: $10.19053 / 20278306.3720$

Carreño-Bodensiek, C. G. (2010). Sistema de control y monitoreo automatizado para gases en minas de carbón. Revista de Investigación, Desarrollo e Innovación, 1 (1), 61-69. Recuperado de: http://revistas.uptc.edu.co/revistas/index.php/in vestigacion_duitama/article/view/1294

Delgado, J., Güell, J., García, J., Conde, M., \& Casado, V. (2014). Aprendizaje de la programación en el Citilab. Revista Iberoamericana de Ciencia Tecnología y Sociedad, 8 (23), 123-133. Recuperado de: http://www.scielo.org.ar/scielo.php?script=sci_ arttext\&pid=S1850 00132014000200008\&lng=es\&tlng=es.

Enríquez-Herrador, R. (2009). Guía de usuario de Arduino. Recuperado de: http://blog.utp.edu.co/librotekdigital/guia-deusuario-de-arduino-rafael-enriquez-herrador/

Fernández-Morales, F. H., \& Duarte, J. E. (2012). Desarrollo de un caudalímetro digital para la medición de caudal de ríos. Revista de Investigación, Desarrollo e Innovación, 3 (1), 44-51. Recuperado de: http://revistas.uptc.edu.co/revistas/index.php/in vestigacion_duitama/article/view/2130

Garcés-Pretel, M., Ruiz-Cantillo, R. \& MartínezAvila, D. (2014). Transformación pedagógica mediada por tecnologías de la información y la comunicación (TIC). Saber, Ciencia y Libertad, 9 (2), 217-228. Recuperado de: https://dialnet.unirioja.es/descarga/articulo/510 4968.pdf 
García-Hurtado, N. D., Castillo-García, L. F., \& Escobar-Jiménez, A. J. (2012). Plataforma robótica educativa "ROBI". Revista Colombiana de Tecnologías de Avanzada, 1 (19), 140-144. Recuperado de: http://revistas.unipamplona.edu.co/ojs_viceinve s/index.php/RCTA/article/view/163

Gausin, S. (2012). Blog spot cómputo integrado. Recuperado de: http://computointegrado.blogspot.com.co/2012/ 04/uso-de-flex-sensor-con-arduino.html

Gutiérrez-Ríos, J., Martínez-Oviedo, E., \& PeñaCortés, C. A. (2013). Desarrollo de un módulo didáctico de robótica paralela y visión artificial con un sistema de sujeción universal. Revista Colombiana de Tecnologías de Avanzada, 1 (21), 74-80. Recuperado de: http://revistas.unipamplona.edu.co/ojs_viceinve s/index.php/RCTA/article/view/299/362

León-Medina, J. X., \& Torres-Barahona, E. A. (2015). Herramienta para el diseño de sistemas de posicionamiento tridimensional usados en fabricación digital. Revista de Investigación, Desarrollo e Innovación, 6 (2), 155-167. http://doi.org/10.19053/20278306.4603

Mesa-Mesa, L. A., \& Barrera-Lombana, N. (2013). La robótica educativa como instrumento didáctico alternativo en educación básica. Revista Colombiana de Tecnologías de Avanzada, 2(22), 59-67. Recuperado de: http://revistas.unipamplona.edu.co/ojs_viceinve s/index.php/RCTA/article/view/411

Monsalves-González, S. (2011). Estudio sobre la utilidad de la robótica educativa desde la perspectiva del docente. Revista de Pedagogía, 32 (90), 81-117. Recuperado de: http://www.redalyc.org/articulo.oa?id=6592005 5004ER.
Moreno, I., Muñoz, L., Serracín, J. R., Quintero, J., Pittí Patiño, K., \& Quiel, J. (2012). La robótica educativa, una herramienta para la enseñanzaaprendizaje de las ciencias y las tecnologías. Revista Teoría de la Educación: Educación y Cultura en la Sociedad de la Información, 13 (2), 74-90. Recuperado de : http://campus.usal.es/ revistas_trabajo/index.p hp/revistatesi/article/view/9000/9245

Reyes-González, D., \& García-Cartagena, Y. (2014). Desarrollo de habilidades científicas en la formación inicial de profesores de ciencias y matemática. Educación y Educadores, 17 (2), 271-285. doi. 10.5294/edu.2014.17.2.4

Parra-León, L. F., Duarte, J. E., \& FernándezMorales, F.H. (2014). Propuesta didáctica para la enseñanza de circuitos eléctricos básicos. Revista de Investigación, Desarrollo e Innovación, 4 (2), 138-147. http://doi.org/10.19053/20278306.2891

Torres-Barahona, E. A., León-Medina, J. X., \& Torres-Díaz, E. (2012). Sistema de posicionamiento aplicado a la técnica de impresión 3D modelado por deposición fundida. Revista de Investigación, Desarrollo e Innovación, 3 (1), 25-32. Recuperado de: http://revistas.uptc.edu.co/revistas/index.php/in vestigacion_duitama/article/view/2135/2091

Velazco-Cáceres, D. F., \& Pinto-Salamanca, M. L. (2012). Caracterización del huevo de gallina para el diseño de un sistema automático de clasificación. Revista de Investigación, Desarrollo e Innovación, 3 (1), 33-43. Recuperado de: http://revistas.uptc.edu.co/revistas/index.php/in vestigacion_duitama/article/view/2136 